\title{
A comparison of relaxation processes in structurally related van der Waals glass formers: The role of internal degrees of freedom
}

\author{
S. Kahle \\ Institut fuer Festkörperforschung, Forschungszentrum Jülich, Postfach 1913, 52425 Jülich, Germany \\ and Max-Planck-Institut für Polymerforschung, Postfach 3148, 55021 Mainz, Germany \\ J. Gapinski \\ Institute of Physics, A. Mickiewicz University, Umultowska 85, 61-614, Poznan, Poland \\ G. Hinze \\ Institut für Physikalische Chemie, Universität Mainz, Jakob-Welderweg 11, 55099 Mainz, Germany \\ A. Patkowski \\ Institute of Physics, A. Mickiewicz University, Umultowska 85, 61-614, Poznan, Poland \\ G. Meier \\ Institut fuer Festkörperforschung, Forschungszentrum Jülich, Postfach 1913, 52425 Jülich, Germany
}

(Received 23 July 2004; accepted 16 November 2004; published online 9 February 2005)

Depolarized dynamic light scattering (DLS), dielectric relaxation (DS), and deuterium NMR studies of fragile van der Waals glass forming liquids phenylphthalein-dimethylether (PDE) and cresolphthalein-dimethylether (KDE) are presented. In PDE a new dielectric loss process was found, which can be attributed to the $180^{\circ}$ flip of the phenyl rings. The previous finding that the distribution of the structural relaxation times measured for PDE and KDE by DS is substantially narrower than that measured by DLS is explained by partial decoupling of the dynamics of the dipole moment from the structural relaxation of the sample. The dynamics of PDE and KDE is compared with the previous studies of two other structurally similar liquids: 1,1'-di(4-methoxy-5methylphenyl)cyclohexane (BMMPC) and 1,1'-bis(p-methoxyphenyl)cyclohexane (BMPC) in order to relate dynamical features with the chemical structure of the material. The evidence for the intramolecular character of the secondary relaxations observed in BMPC and PDE is presented. (C) 2005 American Institute of Physics. [DOI: 10.1063/1.1846653]

\section{INTRODUCTION}

Dynamic processes in supercooled liquids can be studied by different experimental methods assessing different properties of the molecules. Dielectric relaxation spectroscopy provides information on the collective dipole reorientation, depolarized dynamic light scattering on the collective molecules reorientation via fluctuations of the optical anisotropy, while the method of deuterium nuclear magnetic resonance is capable of giving insight into the time scale and the mechanism of a local motion. These various probes (dipole moment, optical anisotropy, etc.), usually localized in different parts of the molecule may couple to a different degree to the dynamics of the entire molecule.

Structural relaxation of a supercooled liquid can be characterized by a primary $(\alpha-)$ relaxation with a non-Arrhenius temperature dependence and at least one secondary $(\beta-)$ relaxation of Arrhenius-type. A typical secondary process is the so-called Johari-Goldstein $(\mathrm{J}-\mathrm{G}) \beta$-relaxation considered as an inherent property of any glass forming system. ${ }^{1}$

The fragile van der Waals liquids investigated here have been extensively studied by several techniques. The temperature dependence of the structural relaxation time has been measured for BMPC and BMMPC by means of DLS and $\mathrm{DS},{ }^{2}$ for PDE using DLS (Ref. 3) and DS (Ref. 4) and for KDE by means of DS (Refs. 4 and 5) in a limited frequency range with a standard resolution. Also the pressure dependence of the $\alpha$-relaxation has been studied by means of DLS and DS for BMMPC (Ref. 6) and PDE (Refs. 3 and 4) and by means of DS for KDE. ${ }^{5}$ The relative volume and temperature contribution to the structural relaxation has been analyzed for BMMPC, BMPC, and PDE by combination of the dynamic and PVT (pressure-volume-temperature) data. ${ }^{7,8}$ BMPC has also been studied by Alvarez et al. ${ }^{9}$ using DS to highlight the region where $\alpha$ - and $\beta$-relaxations merge. Our previous studies on BMMPC by means of positron annihilation ${ }^{10}$ in a very broad temperature range of $20-360 \mathrm{~K}$ have indicated the occurrence of fast relaxation processes in this sample much below $T_{g}$.

In this work we compare dynamical data obtained for two pairs of van der Waals glass forming liquids: BMMPC, BMPC and KDE, PDE (Table I) studied by means of DS, DLS and deuterium solid state NMR. In the previous paper on BMPC and BMMPC it has been shown that the differences in the dynamic behavior of these liquids can be directly related to the differences in their structure. ${ }^{2}$ Because of structural similarities of these materials with PDE and KDE, they are all very well suited for studies aiming at the understanding the relationship between structure modification and relaxational processes on the molecular level.

A supercooled liquid can be characterized by means of fragility defined as the slope of the normalized activation 
TABLE I. Chemical structure, molecular weight $(\mathrm{M})$, melting temperature $\left(T_{m}\right)$, and glass transition temperature $\left(T_{g}\right)$ of the materials used in the study.

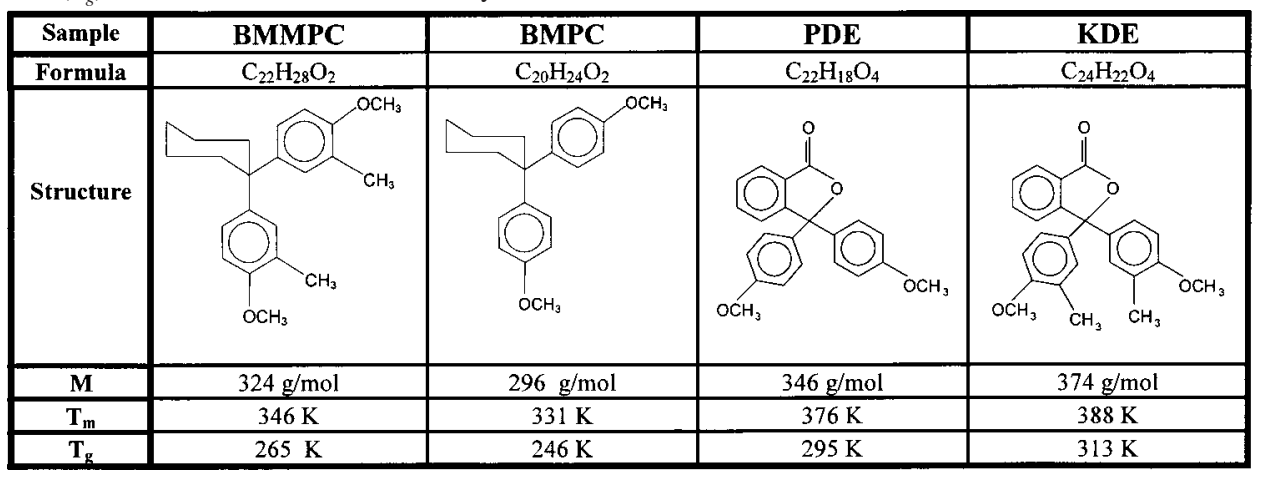

plot at $T_{g}: m=d(\log \tau) / d\left(T_{g} / T\right)$. It has been shown ${ }^{11}$ that for glass forming liquids this fragility is correlated with the nonexponentiality of the correlation function, expressed by the $\beta_{\mathrm{KWw}}$ exponent of the Kohlrausch-Williams-Watts correlation function [Eq. (4)], i.e., fragile liquids are usually characterized by a low $\beta_{\mathrm{KWw}}$ (high nonexponentiality). This correlation seemed to be violated by KDE and PDE, for which in a DS experiment high fragility and low nonexponentiality (high $\beta_{\mathrm{KWW}}$ ) have been observed. ${ }^{4,5}$ In our DLS studies ${ }^{7}$ we have shown that the correlation between $m$ and $\beta_{\mathrm{KWw}}$ holds for the DLS data. We have concluded that the $\beta_{\mathrm{KWW}}$ obtained from DS studies does not describe properly the dynamics of the entire PDE molecules, leading to an apparent disagreement with the general correlation between $m$ and $\beta_{\mathrm{Kww}}$. Among others, the above results have prompted us to look for possible reasons for this discrepancy on the basis of as large as possible comparative study on structurally related van der Waals glass forming liquids.

Therefore, we present in this work DS data obtained for PDE and KDE in extended frequency and sensitivity ranges, as well as deuterium NMR measurements of these two liquids and DLS measurements of KDE in a broad temperature range.The problems we address here are: (i) How does the relaxation spectrum depend on the methods and probes used in a particular experiment and what can we learn about the dynamics of these systems on a molecular level from the comparison of the DS, DLS, and NMR data, (ii) what is the physical origin of the secondary relaxations observed in BMPC and PDE-is this a general J-G relaxation or is it simply internal dynamics characteristic for these molecules, and (iii) what is the relationship between the dynamic behavior and chemical structure of these liquids?

\section{EXPERIMENT}

\section{A. Dielectric spectroscopy (DS)}

Dielectric measurements were carried out using two different setups to cover a broad frequency range from $10^{-2}$ to $2 \times 10^{9} \mathrm{~Hz}$. Isothermal frequency scans were performed in the temperature range from -160 to $+150{ }^{\circ} \mathrm{C}$. A broad band dielectric spectrometer Novocontrol BDS 4000 (based on the high resolution ALPHA Analyzer) was used to measure the dielectric function $\varepsilon^{*}(\nu)=\varepsilon^{\prime}(\omega)-i \varepsilon^{\prime \prime}(\nu)$ at 10 points per frequency decade in a frequency range of $10^{-2}-10^{7} \mathrm{~Hz}$. The sample capacitor consisted of two parallel gild brass-plates with a diameter of $20 \mathrm{~mm}$, separated by the sample. The plate distance of $0.1 \mathrm{~mm}$ was kept constant by small Teflon spacers.

The high frequency range $\left(10^{6}-2 \times 10^{9} \mathrm{~Hz}\right)$ was covered by a Novocontrol BDS 6000 instrument (based on a Hewlett Packard 4291 impedance analyzer). Using the nonlinear least-square Levenberg-Marquard method, the relaxation processes were fitted by a superposition of two Havriliak-Negami (HN) functions $(k=2),{ }^{12}$

$$
\varepsilon^{*}(\omega)=\varepsilon_{\infty}+\sum_{k} \frac{\Delta \varepsilon_{k}}{\left(1+\left(i \omega \tau_{k}\right)^{\alpha_{k}}\right)^{\gamma_{k}}}
$$

with $\omega=2 \pi \nu, \varepsilon_{\infty}$ being the high frequency limit of $\varepsilon^{\prime}(\omega)$ outside the dispersion zone, $\Delta \varepsilon$ the intensities of each process and $0<\alpha \leqslant 1,0<\gamma \leqslant 1$. The maximum loss frequency $\omega_{\max }$ for each process is given by ${ }^{13}$

$$
\omega_{\max }=\frac{1}{\tau}\left(\frac{\sin [\pi \alpha / 2]}{\tan [\pi \alpha / 2(\gamma+1)]}-\cos [\pi \alpha / 2]\right)^{-1 / \alpha} .
$$

For $\omega \ll \omega_{\max }$ and $\omega \gg w_{\max }$ the HN function for $\varepsilon^{\prime \prime}(\omega)$ reduces to power laws with the exponents $\alpha$ and $\alpha \gamma$, respectively. To fit the $\alpha$-process, all parameters were free, while for the intermediate and fast secondary or $\beta$-processes the asymmetry parameter $\gamma$ was set to 1 (corresponds to a symmetric peak which is typical for the $\beta$-process).

\section{B. ${ }^{2} \mathrm{H}-\mathrm{NMR}$ experiment}

The measurements were performed using a home built pulsed ${ }^{2} \mathrm{H}-\mathrm{NMR}$ spectrometer at a deuteron resonance frequency of $55 \mathrm{MHz}$. Typical $\pi / 2$ pulse lengths of about $2.2-$ $2.6 \mu$ s were short enough to stimulate the whole solid state powder spectra. A solid echo pulse sequence $\pi / 2_{x^{-}} \tau-\pi / 2_{y}$ succeeding a saturation pulse sequence was used to record the spectra. To minimize relaxation effects a pulse interval of $\tau=15 \mu$ s was used, however, the finite receiver dead time prevented choosing shorter times. Sample thermalization was realized by using an Oxford Instruments flow cryostat, enabling a temperature stability of $\pm 0.2 \mathrm{~K}$.

\section{Dynamic light scattering (DLS)}

The dynamic depolarized light scattering-photon correlation spectroscopy (PCS) measurements were performed using an argon ion laser operating at $\lambda=514.5 \mathrm{~nm}$ with power 
up to $400 \mathrm{~mW}$. The laser incident polarization was vertical (V) with respect to the scattering plane, whereas the scattered light was detected horizontally $(\mathrm{H})$ resulting in the VH scattering geometry which measured the orientation correlations within our sample in the time range of a PCS experiment. ${ }^{14}$ The scattered light was detected by an avalanche diode detector and an ALV 5000 digital correlator was used to measure the correlation function. In a dynamic light scattering photon correlation spectroscopy experiment the intensity time autocorrelation function $G^{(2)}(t)=\langle I(0) I(t)\rangle$ was measured. This quantity is related to the autocorrelation function of the electric field $g^{(1)}(t)=\langle E(0) E(t)\rangle /\left\langle E(0)^{2}\right\rangle$ through the Siegert relation ${ }^{15}$

$$
G^{2}(t)=\langle I\rangle^{2}\left(1+f\left|g^{(1)}(t)\right|^{2}\right),
$$

where $f$ is a constant. It is a well known fact that the time autocorrelation function for many glass forming systems decays in a nonexponential way. It was experimentally found that the field autocorrelation function was well represented by the empirical Kohlrausch-Williams-Watts (KWW) function given by ${ }^{1}$

$$
g^{(1)}(t)=\exp \left(-\left(\frac{t}{\tau_{\mathrm{KWW}}}\right)^{\beta_{\mathrm{KWW}}}\right),
$$

where $\beta_{\mathrm{KWW}}$ is the KWW parameter ranging between 0 $<\beta \leqslant 1$. The KWW function was also found to describe well our DLS data for KDE, which had not been measured by light scattering photon correlation so far. Fitting Eq. (4) to the experimental correlation functions we obtained the values of $\beta_{\mathrm{KWw}}$ and $\tau_{\mathrm{KWw}}$. From these values the mean relaxation time $\left\langle\tau_{\mathrm{KWW}}\right\rangle$ was calculated:

$$
\left\langle\tau_{\mathrm{KWW}}\right\rangle=\left(\frac{\tau_{\mathrm{KWW}}}{\beta_{\mathrm{KWW}}}\right) \Gamma\left(\beta_{\mathrm{KWW}}^{-1}\right),
$$

where $\Gamma$ denotes the gamma function of $1 / \beta_{\mathrm{KWw}}$.

\section{Differential scanning calorimetry (DSC)}

We measured the glass transition temperature $T_{g}$ using differential scanning calorimetry (DSC). Prior to the $T_{g}$ measurement a few milligrams of each material of all four samples were heated in the aluminum container of the DSC machine (Pyris Diamont, Perkin Elmer) well above the melting temperature and quenched afterwards as fast as possible. Then the samples were heated at $10 \mathrm{~K} / \mathrm{min}$ to find the glass transition temperature, which is also listed in Table I.

\section{E. Samples}

In this study we used phenylphthalein-dimethylether (PDE) and cresolphthalein-dimethylether (KDE). Both substances were synthesized in the laboratory of Professor $\mathrm{H}$. Sillescu at the Johannes Gutenberg University in Mainz, Germany. The chemical structures of both samples are shown together with those of the structurally related BMPC [in earlier papers named BCDE (Ref. 2)] and BMMPC [in earlier papers named BKDE (Ref. 2)] in Table I. For the deuterium NMR experiment we used selectively deuterated PDE- $d_{2}$ and $\mathrm{KDE}-d_{2}$. The samples were prepared by Herbert Zimmer-

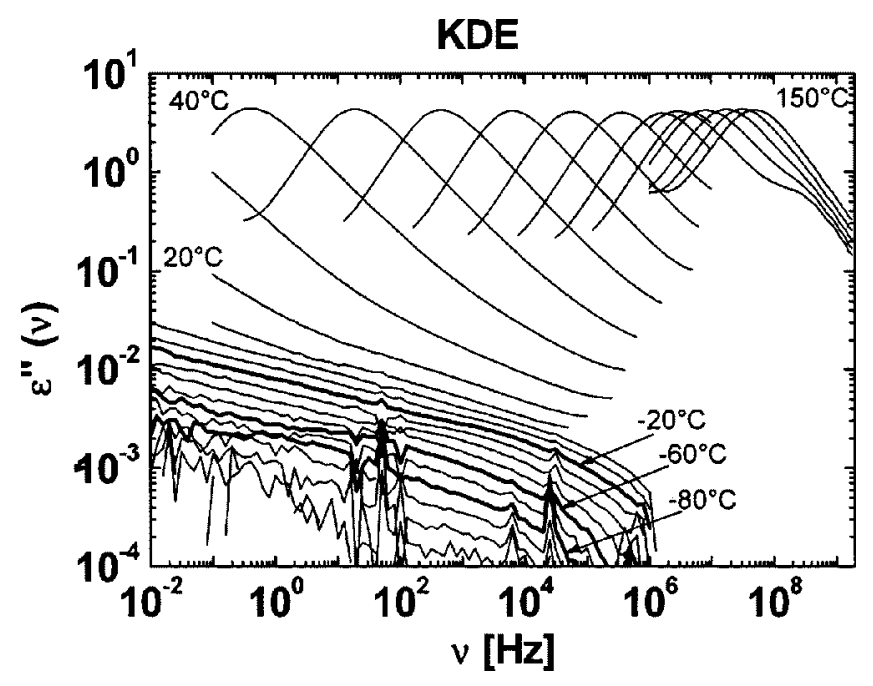

FIG. 1. DS spectra $\varepsilon^{\prime \prime}(\nu)$ vs $\nu$ measured for KDE in the temperature range from -120 to $150{ }^{\circ} \mathrm{C}$ in incremental steps of $\Delta T=10 \mathrm{~K}$. Note that $\varepsilon^{\prime \prime}(\nu)$ goes down to $10^{-4}$ in $\varepsilon^{\prime \prime}(\nu)$. No additional loss processes besides the $\alpha$-relaxation peak are visible at low temperatures, as exemplified for three temperatures (thicker lines).

mann, Heidelberg, Germany. Both samples were deuterated at the phenyl rings in one of the ortho positions to the methoxy groups, hence each molecule contained two deuterium atoms.

\section{RESULTS}

We have performed an extensive dielectric relaxation study on PDE and KDE in the temperature range from -100 to $150{ }^{\circ} \mathrm{C}$ and from -120 to $150{ }^{\circ} \mathrm{C}$, respectively, with an increment of $5 \mathrm{~K}$. The best resolution of our measurements in $\tan (\delta)$ was about $3 \times 10^{-5}$. The data are shown in Figs. 1 and 2 for KDE and PDE, respectively. From inspection of the plots it is evident that for KDE basically two loss processes are observed in contrast to PDE where we find three processes. The processes in KDE are the $\alpha$-relaxation process

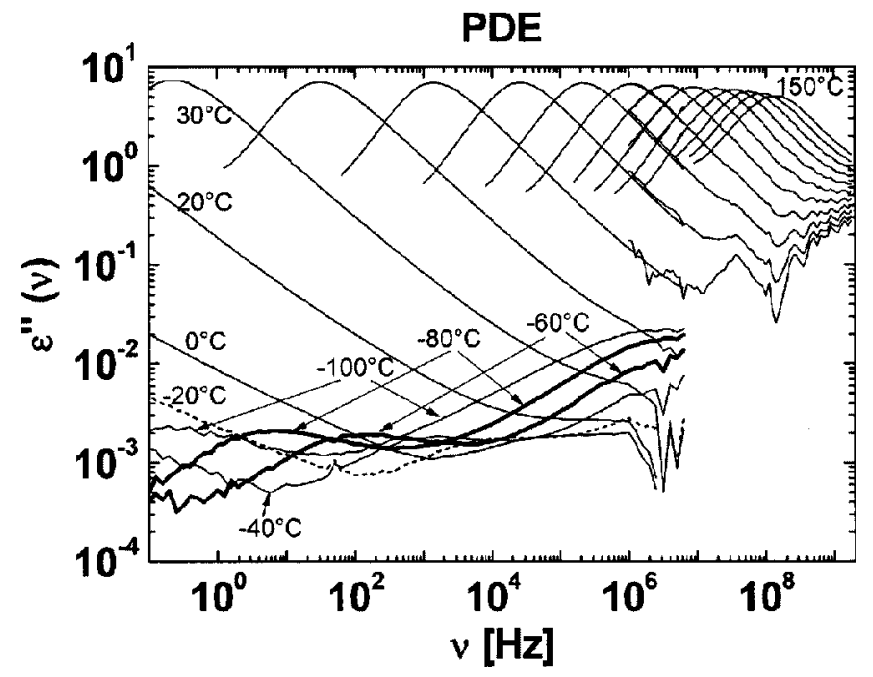

FIG. 2. DS spectra $\varepsilon^{\prime \prime}(\nu)$ vs $\nu$ measured for PDE in the temperature range from -100 to $0{ }^{\circ} \mathrm{C}$ in incremental steps of $\Delta T=20 \mathrm{~K}$ and from 20 to $150{ }^{\circ} \mathrm{C}$ in incremental steps of $\Delta T=10 \mathrm{~K}$. Contrary to KDE shown in Fig. 1, clearly two additional loss processes are visible at low temperatures, as exemplified for three temperatures (thicker lines). 


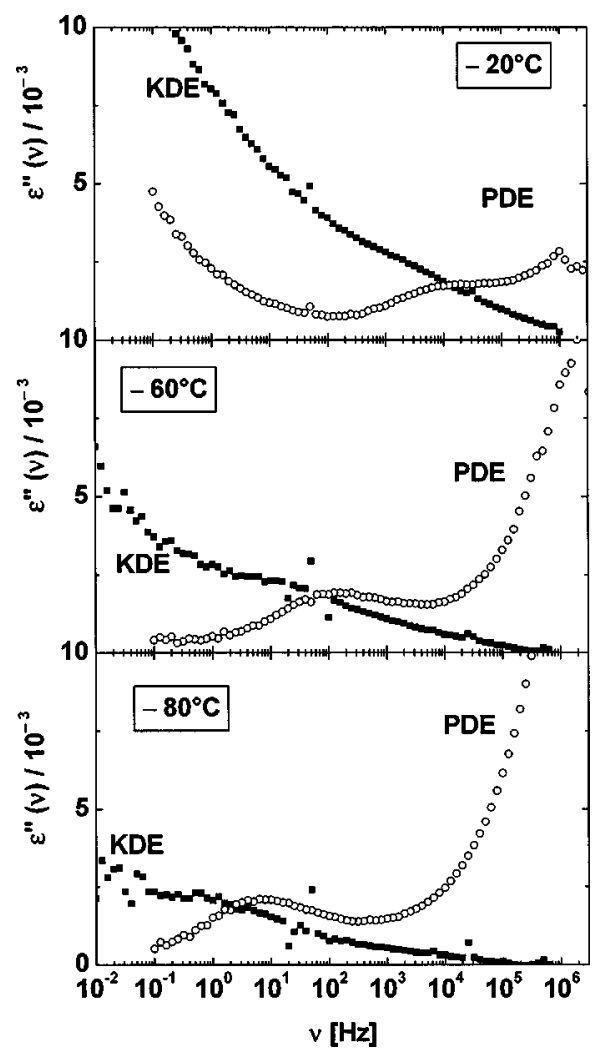

FIG. 3. Comparison of dielectric loss curves measured for PDE and KDE at some selected temperatures outlined also in Figs. 1 and 2 in the region where the phenyl flip occurs. The absence of a pronounced loss peak in $\mathrm{KDE}$ is clearly visible contrary to PDE.

related to the glass transition and additionally a secondary relaxation processes with very low amplitude which can be seen only after a thorough investigation of the low temperature range. In order to show that more clearly, we have chosen three representative low temperatures: $-20,-60$, and $-80^{\circ} \mathrm{C}$ and compared in Figs. 3, the $\varepsilon^{\prime \prime}(\nu)$ data for both materials on a magnified scale.

Whereas for PDE there is a pronounced peak which position shifts from $10 \mathrm{~Hz}$ to about $10^{4} \mathrm{~Hz}$ in the temperature range shown in Fig. 3, there is only a tiny hump seen for KDE. Due to limitations in resolution and the strong influence of the $\alpha$-process it is not possible to analyze this peak in detail. We restrict our analysis to the temperature dependence of the maximum only. This DS-result will be discussed together with the NMR data later in the framework of the Arrhenius diagram, see Fig. 6.

We have analyzed the $\alpha$-relaxation with regard to its width and asymmetry by fitting a Havriliak-Negami function [Eq. (1)] to the $\varepsilon^{\prime \prime}(\omega)$-data and found (the results are listed in Table II for PDE and in Table III for KDE) that the parameter $\alpha$ is about one in the whole temperature range studied in agreement with Stickel et al., ${ }^{4}$ whereas the $\gamma$ parameter increases with increasing temperature also in quantitative agreement with earlier findings. A rather different situation can be found in PDE, where three distinct relaxation processes occur, as seen from the dielectric loss spectra in Fig. 2. The dominant process which appears at the lowest frequency is attributed to the $\alpha$-relaxation. Other, secondary processes appear at higher frequencies and are referred to as $\beta$ - and fast $\beta$-relaxations, respectively. In Figs. 4(a) and 4(b) two additional relaxation processes besides the $\alpha$-relaxation are shown for PDE, at some selected temperatures. Figure 4(a) shows the newly observed weak intermediate loss peak and Fig. 4(b) the high frequency peak corresponding to the process already reported by Stickel et al. ${ }^{4}$ We have found this intermediate process in glassy PDE, where it is visible as a small loss peak on the high frequency side of the spectra (Figs. 2 and 3) and could be observed due to better resolution of our instrument.

In analogy to the hitherto published data on BMPC and BMMPC, ${ }^{2}$ we will present arguments indicating that this new loss process is due to the phenyl ring flip in PDE similarly to the one in BMPC, which is a consequence of the structural similarities between these two materials (see Table I) with regard to the methoxylated phenyl rings. Both substances possess phenyl rings attached to the phthaleinic part of the respective molecules which can rotate on a different time scale than the entire molecule. Such localized motions as a $180^{\circ}$ phenyl flip do not change the optical anisotropy and thus cannot be detected in depolarized dynamic light scattering measurements. Since the process has typical correlation times of the order of milliseconds, it cannot be studied by incoherent quasielastic neutron scattering, which is feasible for faster dynamics. ${ }^{16}$ In order to prove that a $180^{\circ}$ phenyl flip causes the additional loss peak in the DS spectra of PDE, ${ }^{2} \mathrm{H}-\mathrm{NMR}$ spectra of partially deuterated PDE and also KDE samples have been measured by means of the NMR methods which can provide us with precise information on local motion on this time scale. Thanks to the proper localization of the deuterium substitution, ${ }^{2} \mathrm{H}-\mathrm{NMR}$ probes only the motion of the aromatic ring and the phenyl flip is a very characteristic kind of motion, well studied and reported in literature. ${ }^{17}$ We find in PDE the so called 2-phase NMR spectra consisting of two components: a fast one $\left(>10^{5}-10^{6} \mathrm{~Hz}\right)$ and a slow one (typically between single $\mathrm{Hz}$ up to about MHz). Typical experimental ${ }^{2} \mathrm{H}$-NMR spectra for PDE at selected temperatures are shown on the left-hand side of Fig. 5(a). On the right-hand side we show the simulated ones. It is assumed that the data measured can be described by superimposing two dynamically different components shown in the upper right corner of Fig. 5(a) for $T=292.9 \mathrm{~K}$, namely a typical broad Pake spectrum, characteristic of the rigid ${ }^{2} \mathrm{H}-\mathrm{C}$ bond and a more narrow component, characteristic of the $180^{\circ}$ phenyl flip. The spectrum shows typical two horns since for the $180^{\circ}$ flip two sites are equally occupied and only if the overall flipping rate exceeds $10^{5}-10^{6} \mathrm{~Hz}$, one narrow spike is seen, as shown here for $T=340 \mathrm{~K}$. The overall agreement between measurement and simulation is satisfactory, which confirms our interpretation that the additional intermediate loss process in PDE as seen in dielectric relaxation spectroscopy is due to the $180^{\circ}$ phenyl flip, similarly as in BMPC. ${ }^{2}$ Contrary, in Fig. 5(b) we show that for KDE there is no indication for the phenyl motion since only the "rigid" Pake spectrum is seen. The observation of a small, tiny hump in the DS-spectra of KDE, as shown in Fig. 3, needs further attention with regard to its possible mechanism. Since the $180^{\circ}$ flip of the phenyl group is ruled out for $\mathrm{KDE}$, it is possibly a small angle jiggling of the phenyl rings, 
TABLE II. Fit parameters of the $\alpha$-relaxation of PDE according to the Havriliak-Negami Eq. (1) (dielectric spectroscopy) and according to the Kohlrausch-Williams-Watts Eq. (4) (dynamic light scattering), respectively. The corresponding $\beta_{\mathrm{KWw}}$ parameter of dielectric spectroscopy was calculated according to Eq. (6).

\begin{tabular}{|c|c|c|c|c|c|c|c|}
\hline \multirow[b]{2}{*}{ Temp $\left({ }^{\circ} \mathrm{C}\right)$} & \multicolumn{5}{|c|}{ DS } & \multicolumn{2}{|c|}{ DLS } \\
\hline & $\alpha_{\mathrm{HN}}$ & $(\alpha \gamma)_{\mathrm{HN}}$ & $\left(\beta_{\mathrm{KWW}}\right)_{\mathrm{DS}}$ & $\tau_{\mathrm{HN}}(\mathrm{s})$ & $1 / \omega_{\max }(\mathrm{s})$ & $\left\langle\tau_{\mathrm{KWW}}\right\rangle_{\mathrm{DLS}}(\mathrm{s})$ & $\left(\beta_{\mathrm{KWW}}\right)_{\mathrm{DLS}}$ \\
\hline 30 & 0.968 & 0.616 & 0.67 & $9.33 \mathrm{E}-1$ & $6.42 \mathrm{E}-1$ & & \\
\hline 35 & 0.968 & 0.648 & 0.70 & $7.00 \mathrm{E}-2$ & $5.00 \mathrm{E}-2$ & & \\
\hline 40 & 0.98 & 0.662 & 0.72 & $7.00 \mathrm{E}-3$ & $5.00 \mathrm{E}-3$ & & \\
\hline 45 & 0.995 & 0.677 & 0.73 & $9.09 \mathrm{E}-4$ & $6.69 \mathrm{E}-4$ & & \\
\hline 50 & 1.009 & 0.694 & 0.74 & $1.49 \mathrm{E}-4$ & $1.11 \mathrm{E}-4$ & & \\
\hline 55 & 1.018 & 0.718 & 0.76 & $3.02 \mathrm{E}-5$ & $2.31 \mathrm{E}-5$ & & \\
\hline 60 & 1.018 & 0.748 & 0.79 & $7.74 \mathrm{E}-6$ & $6.12 \mathrm{E}-6$ & & \\
\hline 65 & 1.019 & 0.776 & 0.81 & $2.38 \mathrm{E}-6$ & $1.93 \mathrm{E}-6$ & & \\
\hline 70 & 1.016 & 0.802 & 0.84 & $8.69 \mathrm{E}-7$ & $7.25 \mathrm{E}-7$ & & \\
\hline 75 & 1.011 & 0.84 & 0.87 & $3.63 \mathrm{E}-7$ & $3.14 \mathrm{E}-7$ & & \\
\hline 80 & 1.016 & 0.815 & 0.85 & $1.82 \mathrm{E}-7$ & $1.54 \mathrm{E}-7$ & & \\
\hline 85 & 1.011 & 0.804 & 0.84 & $9.62 \mathrm{E}-8$ & $8.06 \mathrm{E}-8$ & & \\
\hline 90 & 1.04 & 0.81 & 0.84 & $4.94 \mathrm{E}-8$ & $4.51 \mathrm{E}-8$ & & \\
\hline 95 & 1.02 & 0.839 & 0.87 & $2.50 \mathrm{E}-8$ & $2.64 \mathrm{E}-8$ & & \\
\hline 100 & 1.016 & 0.844 & 0.87 & $2.01 \mathrm{E}-8$ & $1.87 \mathrm{E}-8$ & & \\
\hline 105 & 1.016 & 0.837 & 0.86 & $1.49 \mathrm{E}-8$ & $1.27 \mathrm{E}-8$ & & \\
\hline 110 & 1.015 & 0.831 & 0.86 & $1.03 \mathrm{E}-8$ & 8.95E-9 & & \\
\hline 115 & 1.017 & 0.817 & 0.85 & $7.76 \mathrm{E}-9$ & $6.66 \mathrm{E}-9$ & & \\
\hline 120 & 1.008 & 0.84 & 0.87 & $5.95 \mathrm{E}-9$ & $5.06 \mathrm{E}-9$ & & \\
\hline 125 & 1.015 & 0.795 & 0.83 & $4.59 \mathrm{E}-9$ & 3.95E-9 & & \\
\hline 130 & 1.016 & 0.781 & 0.82 & $3.75 \mathrm{E}-9$ & $3.18 \mathrm{E}-9$ & & \\
\hline 135 & 1.017 & 0.759 & 0.80 & $3.25 \mathrm{E}-9$ & 2.64E-9 & & \\
\hline 140 & 1.005 & 0.816 & 0.85 & $2.81 \mathrm{E}-9$ & $2.21 \mathrm{E}-9$ & & \\
\hline 145 & 1.002 & 0.824 & 0.85 & $2.37 \mathrm{E}-9$ & $1.89 \mathrm{E}-9$ & & \\
\hline 150 & 0.994 & 0.843 & 0.87 & $1.97 \mathrm{E}-9$ & $1.61 \mathrm{E}-9$ & & \\
\hline 23.5 & & & & & & 51.7 & 0.54 \\
\hline 24.5 & & & & & & 27 & 0.53 \\
\hline 26.4 & & & & & & 5.7 & 0.56 \\
\hline 27.4 & & & & & & 3.7 & 0.54 \\
\hline 29.3 & & & & & & 1.4 & 0.53 \\
\hline 31.3 & & & & & & 0.50 & 0.53 \\
\hline 33.3 & & & & & & 0.17 & 0.55 \\
\hline 35.2 & & & & & & 0.070 & 0.54 \\
\hline 37.2 & & & & & & 0.024 & 0.57 \\
\hline 39.0 & & & & & & 0.0099 & 0.55 \\
\hline 41.1 & & & & & & 0.0040 & 0.57 \\
\hline 45.0 & & & & & & 0.00087 & 0.57 \\
\hline 43.9 & & & & & & 0.0013 & 0.56 \\
\hline 48.9 & & & & & & 0.00019 & 0.59 \\
\hline 52.8 & & & & & & 0.000048 & 0.61 \\
\hline 56.7 & & & & & & 0.000012 & 0.60 \\
\hline
\end{tabular}

which may cause this small effect. This is not in contradiction to the NMR result, since it is very difficult to detect a small angle motion by this method. Here further measurements are in progress which will be taken at various temperatures in order to investigate changes in the width of the spectra which are indicative of small angle motion.

We have analyzed the relaxation modes presented in Figs. 1 and 2 with regard to the temperature dependence of the positions of their maxima as derived from the fit of the Havriliak-Negami function to the experimental data and have plotted the results in Fig. 6 in an Arrhenius representation. Several important features emerge from this figure. The relaxation times of the $\alpha$-processes show the typical non-
Arrhenius temperature behavior. Adopting a typical relaxation time of $100 \mathrm{~s}$ at the glass transition, different $\alpha$-traces for both specimens reflect their different glass transition temperatures as is depicted in Table I.

It has been shown that for the majority of nonpolymeric glass forming systems, including the van der Waals glass forming systems studied here, the low frequency flank of the relaxation time distribution is characterized by a slope of about one (it corresponds to $\alpha=1$, Table II and III) in contrast to those for polymeric systems, whereas the high frequency wing is described by the slope $-\alpha \gamma$. Our values for $\alpha$ and $\gamma$ fit well to those published by Stickel et al. ${ }^{4}$ Due to this asymmetric relaxation time distribution, the Kohlrausch- 
TABLE III. Fit parameters of the $\alpha$-relaxation of KDE according to the Havriliak-Negami Eq. (1) (dielectric spectroscopy) and according to the Kohlrausch-Williams-Watts Eq. (4) (dynamic light scattering), respectively. The corresponding $\beta_{\mathrm{KWw}}$ parameter of dielectric spectroscopy was calculated according to Eq. (6).

\begin{tabular}{|c|c|c|c|c|c|c|c|}
\hline \multirow[b]{2}{*}{ Temp $\left({ }^{\circ} \mathrm{C}\right)$} & \multicolumn{5}{|c|}{ DS } & \multicolumn{2}{|c|}{ DLS } \\
\hline & $\alpha_{\mathrm{HN}}$ & $(\alpha \gamma)_{\mathrm{HN}}$ & $\left(\beta_{\mathrm{KWW}}\right)_{\mathrm{DS}}$ & $\tau_{\mathrm{HN}}(\mathrm{s})$ & $1 / \omega_{\max }(\mathrm{s})$ & $\left\langle\tau_{\mathrm{KWW}}\right\rangle_{\mathrm{DLS}}(\mathrm{s})$ & $\left(\beta_{\mathrm{KWW}}\right)_{\mathrm{DLS}}$ \\
\hline 45 & & & & & & 2.15076 & 0.515 \\
\hline 50 & 1 & 0.63 & 0.687 & $5.600 \mathrm{E}-1$ & $3.89 \mathrm{E}-1$ & 0.24928 & 0.537 \\
\hline 55 & 1 & 0.655 & 0.709 & $7.233 \mathrm{E}-2$ & $5.18 \mathrm{E}-2$ & 0.03677 & 0.555 \\
\hline 60 & 0.992 & 0.678 & 0.729 & $1.121 \mathrm{E}-3$ & $8.28 \mathrm{E}-3$ & 0.00721 & 0.572 \\
\hline 65 & 1.005 & 0.693 & 0.742 & $2.130 \mathrm{E}-3$ & $1.59 \mathrm{E}-3$ & 0.00138 & 0.585 \\
\hline 70 & 1.008 & 0.712 & 0.759 & $4.566 \mathrm{E}-4$ & $3.48 \mathrm{E}-4$ & $2.86 \mathrm{E}-4$ & 0.599 \\
\hline 75 & 1.013 & 0.73 & 0.774 & $1.132 \mathrm{E}-5$ & $8.79 \mathrm{E}-5$ & $6.35 \mathrm{E}-5$ & 0.62 \\
\hline 80 & 1.016 & 0.744 & 0.786 & $3.157 \mathrm{E}-5$ & $2.49 \mathrm{E}-5$ & $1.32 \mathrm{E}-5$ & 0.633 \\
\hline 83 & & & & & & $7.51 \mathrm{E}-6$ & 0.64 \\
\hline 85 & 1.015 & 0.758 & 0.798 & $9.739 \mathrm{E}-6$ & $7.78 \mathrm{E}-6$ & & \\
\hline 90 & 1.015 & 0.773 & 0.811 & $3.321 \mathrm{E}-6$ & $2.69 \mathrm{E}-6$ & & \\
\hline 95 & 1.014 & 0.787 & 0.823 & $1.245 \mathrm{E}-6$ & $1.02 \mathrm{E}-6$ & & \\
\hline 100 & 1.011 & 0.799 & 0.833 & $5.165 \mathrm{E}-7$ & $4.31 \mathrm{E}-7$ & & \\
\hline 105 & 1.011 & 0.796 & 0.831 & $2.358 \mathrm{E}-7$ & $1.96 \mathrm{E}-7$ & & \\
\hline 110 & 0.925 & 0.784 & 0.820 & $5.322 \mathrm{E}-8$ & $9.64 \mathrm{E}-8$ & & \\
\hline 115 & 0.961 & 0.775 & 0.813 & $3.478 \mathrm{E}-8$ & $4.82 \mathrm{E}-8$ & & \\
\hline 120 & 0.979 & 0.748 & 0.790 & $1.676 \mathrm{E}-8$ & $3.28 \mathrm{E}-8$ & & \\
\hline 125 & 0.986 & 0.722 & 0.767 & $1.130 \mathrm{E}-8$ & $1.30 \mathrm{E}-8$ & & \\
\hline 130 & 1.004 & 0.759 & 0.799 & $7.960 \mathrm{E}-9$ & $9.08 \mathrm{E}$ & & \\
\hline 135 & 1.005 & 0.8 & 0.834 & $5.745 \mathrm{E}-9$ & $6.66 \mathrm{E}$ & & \\
\hline 140 & 1.002 & 0.846 & 0.873 & $4.403 \mathrm{E}-9$ & $5.03 \mathrm{E}$ & & \\
\hline 145 & 1.002 & 0.879 & 0.900 & $3.451 \mathrm{E}-9$ & $3.97 \mathrm{E}$ & & \\
\hline 150 & 1.002 & 0.903 & 0.920 & $5.600 \mathrm{E}-9$ & $3.18 \mathrm{E}$ & & \\
\hline
\end{tabular}

Williams-Watts function in its Fourier transformed form makes usually a good approximation of the dielectric spectrum as shown by Boese et al. ${ }^{18}$ This is further exemplified by the depolarized dynamic light scattering results obtained
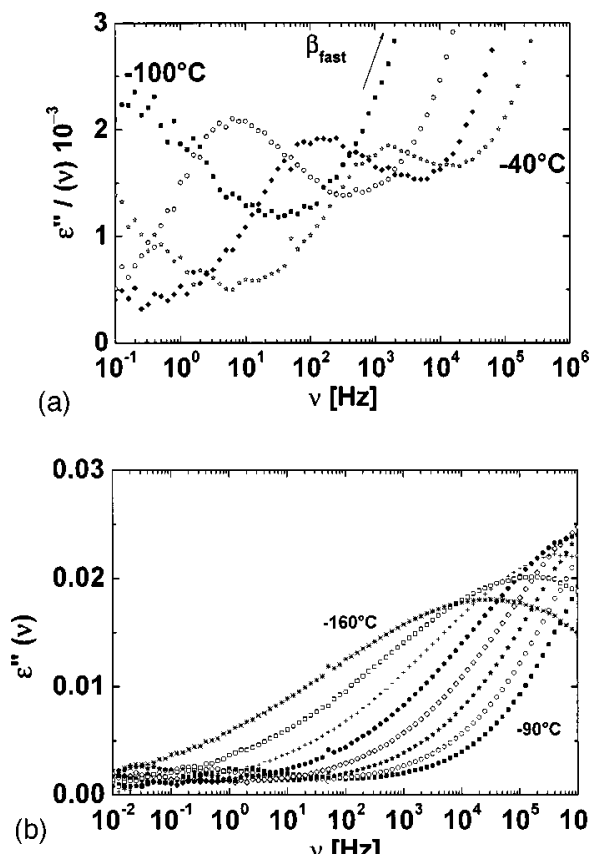

FIG. 4. (a) $\varepsilon^{\prime \prime}(\nu)$ vs $\nu$ for PDE in the region of the phenyl flip motion measured at $-100(\mathbf{\square}),-80(\bigcirc),-60(\diamond)$, and $-40{ }^{\circ} \mathrm{C}\left(\right.$ 污). (b) $\varepsilon^{\prime \prime}(\nu)$ vs $\nu$ for PDE in the region of the fast $\beta$-process measured in the temperature range from -160 to $-90{ }^{\circ} \mathrm{C}$ in incremental steps of $\Delta T=10 \mathrm{~K}$. for KDE above $T_{g}$. These data are shown in Fig. 7 for some selected temperatures together with the results of the fit of Eq. (4) to the data. The values of $\beta_{\mathrm{KWw}}$ and the mean relaxation time $\langle\tau\rangle$ for different temperatures calculated using Eq. (5) are listed in Table III. In order to compare the parameters obtained in the time domain by means of DLS with those
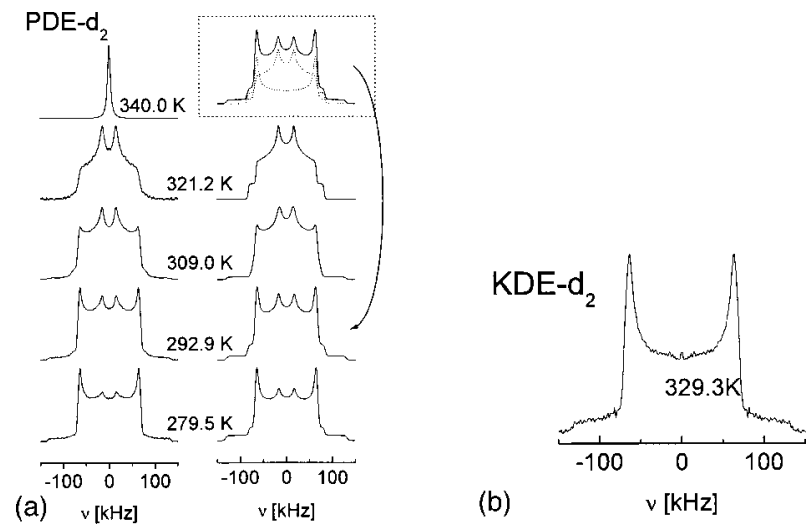

FIG. 5. (a) Comparison between experimentally determined ${ }^{2} \mathrm{H}-\mathrm{NMR}$ spectra obtained for PDE- $d_{2}$ at some selected temperatures as indicated in the plot (left-hand side of the figure) with simulated data (right-hand side of the figure). In the upper right corner the decomposition of a spectrum is shown as an example for the separation into the rigid Pake part and the contribution due to phenyl flip motion. Note that at $T=340 \mathrm{~K}\left(=77^{\circ} \mathrm{C}\right)$, we observe only one narrow component due to motional narrowing, which means that the motion is faster than the inverse of the quadrupolar coupling constant of about $250 \mathrm{kHz}$. (b) ${ }^{2} \mathrm{H}-\mathrm{NMR}$ spectra obtained for KDE- $d_{2}$ at $T=329.3 \mathrm{~K}$ $\left(\cong 56{ }^{\circ} \mathrm{C}\right)$. Here only a rigid Pake spectrum is visible and no indication for a phenyl flip shows up. The same holds also for other temperatures in the glassy state of the material. 


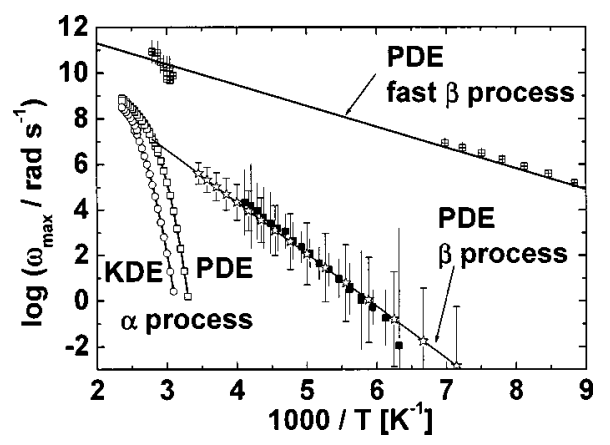

FIG. 6. Arrhenius plot of the relaxation frequencies of processes observed in KDE (O) and PDE ( $\square$ ) by means of DS. The $\omega_{\max }$ values were calculated using Eq. (2). The notation of the $\beta$-processes refers only to PDE: The fast $\beta$-process $(\boxplus)$, which has been already reported by Stickel et al. (Ref. 4) and the new $\beta$-process ( - DS and - NMR), which stems from the phenyl flip motion. The NMR correlation times were calculated from the spectra following a procedure described by Roessler et al. (Ref. 20).

from DS obtained in the frequency domain one data set has to be Fourier transformed. Exact numerical recipes are available to correlate the Havriliak-Negami $\alpha$ and $\gamma$ parameters with the $\beta_{\mathrm{KWw}}$ parameter. We chose the approximation given by Alvarez et al. ${ }^{19}$ which simply reads

$$
\beta_{\mathrm{KWW}}=(\alpha \gamma)^{1 / 1.23} \text {. }
$$

For BMPC a good agreement was found between the $\beta_{\mathrm{KWw}}$ parameters derived from dynamic light scattering and dielectric relaxation, both yielded $\beta_{\mathrm{KWw}}$ values about 0.5 in the temperature range between -23 and $25^{\circ} \mathrm{C}$. For PDE, however, Patkowski et al. ${ }^{3}$ report a large difference between $\beta_{\mathrm{KWw}}$ from light scattering, being about $0.5-0.6$ in the temperature range between 20 and $60{ }^{\circ} \mathrm{C}$, and the value for $\beta_{\mathrm{KWw}}$ from dielectric relaxation, which lies between 0.7 and $0.8^{4}$ in a similar temperature range. In Fig. 8 we show the $\beta_{\mathrm{KWw}}$ parameters of PDE and KDE as a function of temperature as obtained from DLS and DS, respectively. For KDE we find the $\beta_{\mathrm{KWw}}$ parameter, obtained from DS, which increases slightly with temperature from 0.7 to 0.85 in the temperature range between 40 and $110{ }^{\circ} \mathrm{C}$ and is thus also markedly higher than the value for $\beta_{\mathrm{KWw}}$ obtained from DLS ranging from 0.5 to 0.63 in the temperature range from 45 to $83^{\circ} \mathrm{C}$ (cf. Table III). This finding is also in disagreement

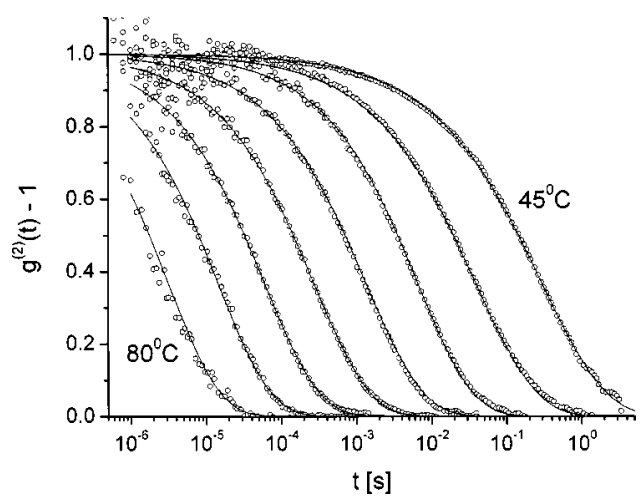

FIG. 7. Normalized intensity autocorrelation functions obtained for KDE in the $\mathrm{VH}$ scattering geometry for temperatures from 45 (right) to $80^{\circ} \mathrm{C}$ (left) with an increment of $\Delta T=5 \mathrm{~K}$. A fit of a KWW function [Eq. (4)] to the data is shown as a solid line.

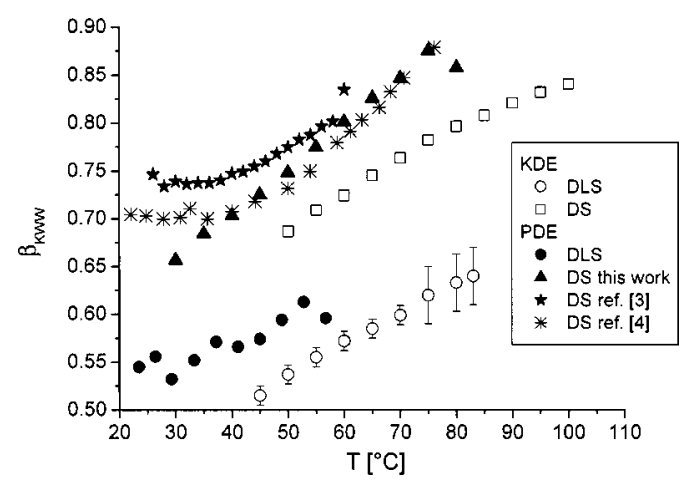

FIG. 8. Comparison of the $\beta_{\mathrm{KWw}}$ parameters obtained by DLS and DS for PDE [DLS (Ref. 3), DS (Ref. 4)] and of KDE [DLS (this work), DS (Ref. 5)]. $\beta_{\mathrm{KWw}}$ parameters for DS were calculated from the HN parameters (Table I) using Eq. (6). For PDE two other DS data sets, besides our own data, are available: Patkowski et al. (Ref. 3) and Stickel (Ref. 4). Our own data agree reasonably well with these, however, there are deviations at low temperatures. They stem from the different handling of the high frequency contributions in the spectra.

with the light scattering and dielectric results obtained for the structurally related BMMPC (in the temperature range between 0 and $\left.100^{\circ} \mathrm{C}\right),{ }^{2,4}$ where a similar $\beta_{\mathrm{KWw}}$ was found by both methods. As already stated, the temperature dependence of the frequency at the maximum, $\omega_{\max }$, depicted in Fig. 6 is highly non-Arrhenius-type and can be routinely expressed in terms of the Vogel-Fulcher-Tammann equation (VFT)

$$
\omega_{\max }=\omega_{0} \exp \left(\frac{-B}{T-T_{0}}\right), \quad\langle\tau\rangle=\left\langle\tau_{0}\right\rangle \exp \left(\frac{B}{T-T_{0}}\right),
$$

with $\omega_{0}$ and $\left\langle\tau_{0}\right\rangle$ being the high temperature limits, respectively. In the case of DS, the data are represented in the frequency domain, whereas for DLS the time representation is used. $B$ is a kind of activation energy related to the fragility of the system ${ }^{1}$ and $T_{0}$ is the Vogel temperature.

In Fig. 9 we plot the $-\log \left(\omega_{\max }\right)$ data from DS and the $\log (\langle\tau\rangle)$ data from DLS versus temperature and find excellent agreement although strictly speaking we do not compare the mean times from the $\mathrm{HN}$ distribution with the mean times from light scattering experiment but use $1 / \omega_{\max }$ instead.

Boese et $a l .{ }^{18}$ have found that the Fourier transformation of DS data and a subsequent fit of the KWW function to that

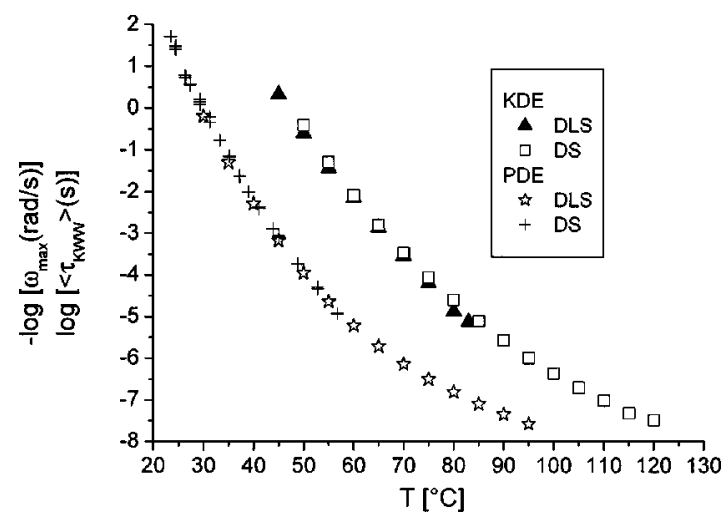

FIG. 9. Comparison of the logarithm of the mean relaxation times $\langle\tau\rangle$ vs temperature for PDE and KDE obtained by DLS and the negative logarithm of the maximum frequency $\omega_{\max }$ from DS. Data from DLS are calculated via Eq. (5) and data from DS are calculated via Eq. (2) using $\omega_{\max }=1 / \tau_{\max }$. 
result yielded $\beta$ parameters identical within $10 \%$ to that obtained from DLS. There is no simple reason why the relaxation time distribution for dipole reorientations should be identical to the orientational correlations monitored via fluctuations in the optical anisotropy. We only want to stress that by plotting our data as shown in Fig. 9 we reach a good agreement between both data sets for both materials. Our VFT parameters for PDE (low temperature region) are $B$ $=1562 \pm 115 \mathrm{~K}$ and $T_{0}=253 \pm 3 \mathrm{~K}$ and those for KDE are $B$ $=3048 \pm 100 \mathrm{~K}$ and $T_{0}=240 \pm 2 \mathrm{~K}$. These VFT parameters for PDE and the crossover temperature from one set of VFT parameters describing the temperature dependence of $\tau_{\alpha}$ at low temperatures to another one at high temperatures are in agreement with the results of Stickel et al. ${ }^{4}$ These parameters (high temperature region) are: for PDE $B=490 \pm 16 \mathrm{~K}$ and $T_{0}=295 \pm 2 \mathrm{~K}$ and for $\mathrm{KDE} \quad B=393 \pm 14 \mathrm{~K}$ and $T_{0}$ $=326 \pm 2 \mathrm{~K}$.

It should be noted that the crossover temperature in KDE at about $T=380 \mathrm{~K}^{4}$, might be related to a merging of the $\alpha$ and $\beta$-processes seen as a weak hump, cf. Fig. 3, which we cannot analyze quantitatively similarly as for PDE, for which the crossover temperature coincides with the merging temperature of the $\alpha$ trace with the phenyl flip motion at about $T=330 \mathrm{~K}$, also in good agreement with Stickel et al. ${ }^{4}$ The relaxation times of the latter mode, which is being addressed to as the $\beta$-process, obtained by means of DS and NMR, are shown in Fig. 6. Both data sets coincide within the error bars thus confirming our earlier conclusion that the molecular origin of the newly observed $\beta$-process in PDE is the phenyl flip motion. Its activation energy amounts to $40 \mathrm{~kJ} / \mathrm{mol}$. The reason why there is no such motion visible in $\mathrm{KDE}$ is that the methyl group in ortho position makes the ring asymmetric; its rotation is no more possible without a cooperative rearrangement of the neighbors.

With regard to the nomenclature, we want to point out that usually the term of the "fast $(\beta-)$ process" is entirely devoted to the so-called rattling-in-the-cage motion which had been first reported from incoherent quasielastic neutron scattering and later also from light scattering studies of glass forming systems. This motion occurs at a frequency of about $10^{11}$ to $10^{12} \mathrm{~Hz}$ and is hence much faster than our fast $\beta$ motion reported here which can be assigned to the rotation of the methoxy group, in analogy to the corresponding process found in BMPC. ${ }^{2}$ The activation energy is in a good agreement with that determined for pure anisol. ${ }^{2}$ The arguments why there is no such motion visible in KDE are similar to those given by Meier $e t a l .^{2}$ for the structurally related material BMMPC: The methyl group in ortho position to the methoxy group prevents the dipole moment of the latter to rotate freely due to an overlap of the van der Waals radii between the methyl protons of the methoxy group with those from the ortho methyl group.

\section{DISCUSSION}

Summarizing our main experimental results and thereby also taking the results for the structurally related BMPC and BMMPC into consideration, we can say that in both pairs of van der Waals glass forming liquids PDE/BMPC and KDE/
BMMPC we find qualitatively very similar activation plots in each of the pairs with the same number of processes: three for PDE and BMPC and only one well developed process for $\mathrm{KDE}$ and BMMPC (in the case of KDE an additional process of very low amplitude is also visible in the DS spectra). We will start our discussion with the secondary relaxations. We could show that in PDE and BMPC we can find a molecular mechanistic explanation of the two secondary relaxation processes. In KDE and BMMPC no secondary relaxations are visible in the ${ }^{2} \mathrm{H}-\mathrm{NMR}$ spectra, however, there are indications of a small angle jiggling of the phenyl groups in $\mathrm{KDE}$ and possibly in BMMPC following from the structural similarities between these two materials. It should be noted that we have tried to find secondary relaxations using DLS in the latter materials, however, without success.

The physical nature of secondary relaxations is a matter of a longstanding controversy between those who claim that these always originate from distinct intramolecular motions and those who believe that it is a universal inherent process independent of the underlying system or, so to speak, of its chemical structure, like the so called Johari-Goldstein process. The secondary relaxations we have measured in PDE do not have the properties of Johari-Goldstein processes, since we find a distinct intramolecular origin of them. Thus, we can conclude that the modes we discuss are not of JohariGoldstein-type. On the other hand, these secondary processes exhibit an Arrhenius temperature dependence, and merge with the $\alpha$-relaxation at some distinguished temperature. We believe that in most cases one can find a molecular origin of any secondary relaxation, however, it is an open question, whether the internal relaxation is connected with the reorientation of the whole molecule or not.

The peculiar finding of different widths of the relaxation time distributions of the $\alpha$-processes obtained by DLS and DS in the pairs PDE-KDE and BMPC-BMMPC needs further consideration. The main difference can be seen in the KWW parameters characterizing the $\alpha$-process. While for BMMPC and BMPC both the relaxation times and $\beta_{\mathrm{KWW}}$ exponents obtained by means of DS and DLS are the same, for the other two molecules $\beta_{\mathrm{KWW}}(\mathrm{DS})>\beta_{\mathrm{KWW}}(\mathrm{DLS})$, Fig. 8. What can be the reason for this difference? In the DS experiment we are monitoring the reorientational dynamics of permanent dipole moments. In PDE and KDE there is a strong dipole moment in the middle of the molecule (about 2 D) which is coupled to the phenyl ring (1), Fig. 10, and two equivalent weaker dipoles (about $0.8 \mathrm{D}$ ) in the side groups $\left(-\mathrm{O}-\mathrm{CH}_{3}\right)$ of rings (2) and (3), Fig. 10. The DS signal is dominated by the contribution coming from the strongest dipole coupled to ring (1).

Thus, is it possible that the DS is monitoring mainly the dynamics of a part of the molecule [strong dipole+ring (1)], which could result in a narrower distribution of relaxation times and a higher $\beta_{\mathrm{KWw}}$ value? KDE and PDE have quite different intramolecular degrees of freedom. While in PDE the $180^{\circ}$ phenyl ring flip can be seen, in KDE merely a small angle jiggling of the rings may be present. However, both substances show similar differences $\beta_{\mathrm{KWw}}(\mathrm{DS})$ 


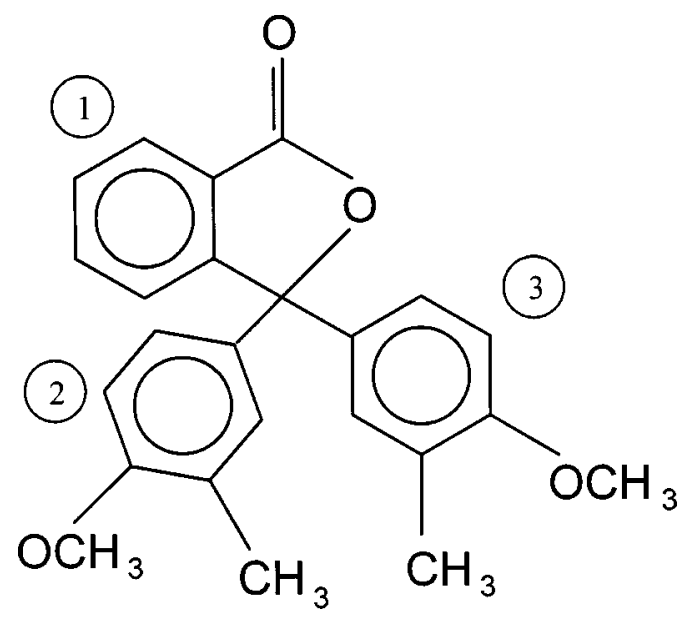

FIG. 10. Molecular structure of KDE. The numbers in circles are meant only to number the rings for a more convenient reference.

$>\beta_{\mathrm{KWW}}(\mathrm{DLS})$ which may indicate that this difference is not related to the presence or absence of the phenyl flip relaxation.

Both techniques DS and DLS probe molecular rotational dynamics. Due to the different ranks $\ell$ of the respective interaction tensors, distinct rotational correlation functions are obtained. That is, for the case of simple isotropic rotational diffusion the resulting time scales could be 3 times faster for DLS $(\ell=2)$ than those obtained by DS $(\ell=1)$. However, in supercooled liquids often similar times have been observed by both experimental techniques. ${ }^{21}$ This has been attributed to a complex relaxation scenario with averaging effect which can almost nullify the differences.

As already mentioned, a significant correlation between the fragility of glass formers and their stretching parameters for the $\alpha$-process has been observed. ${ }^{11}$ Since PDE and KDE are quite fragile glass formers, our observed $\beta_{\mathrm{KWw}}(\mathrm{DLS})$ values fit to this correlation while the $\beta_{\mathrm{KWW}}(\mathrm{DS})$ values are somehow too large. An important difference between the pairs PDE-KDE and BMPC-BMMPC is the presence of an additional phtalic group with dominating permanent dipole moment in PDE-KDE. For PDE-KDE the differences in the stretching parameters $\beta_{\mathrm{KWW}}(\mathrm{DS})>\beta_{\mathrm{KWW}}(\mathrm{DLS})$ may be somehow related to the partial decoupling of the dynamics of the dipole moment from the structural relaxation of the sample as has been observed in some alcohols. ${ }^{22-24}$ For BMMPC and BMPC such decoupling is not possible and $\beta_{\mathrm{KWW}}(\mathrm{DS})=\beta_{\mathrm{KWW}}(\mathrm{DLS})$ because both the dipole moment and the optical anisotropy are coupled to the aromatic rings. The central part of these molecules contains a cyclohexyl ring having no dipole moment and very low optical anisotropy, thus contributing very little to the measured signals. Therefore, both DS and DLS monitor the same dynamics.

\section{CONCLUSIONS}

Dynamics of PDE and KDE has been studied in broad temperature and frequency ranges by means of DS, deuterium NMR and DLS. In PDE, in addition to the usual $\alpha$-relaxation, two Arrhenius-type secondary $(\beta-)$ processes have been observed and assigned to the internal relaxations: a newly observed process assigned to the phenyl flip and a fast process due to reorientation of the $-\mathrm{OCH}_{3}$ group.

Only a very weak secondary relaxation has been observed in KDE. This is due to the presence of additional $-\mathrm{CH}_{3}$ groups on both rings in the ortho position to the methoxy group, which makes the $180^{\circ}$ phenyl impossible due to the spatial hindrance. There are possibly small angle jigglings present which need further experimental investigations. Thus, for PDE and KDE one can see a clear relationship between the chemical structure and the relaxation pattern, similar to that observed for BMPC and BMMPC.

The fact that the width of the distribution of the structural relaxation times measured for KDE and PDE by DS is narrower than that obtained from DLS is attributed to the partial decoupling of the dynamics of the permanent dipole moments in PDE-KDE from the structural relaxation of the sample. While the detailed mechanism remains still unclear, we refer to a similar effect found in some supercooled alcohols. For the chemically similar molecules BMPC and BMMPC no such differences have been observed since in this case both the dipole moment (DS) and optical anisotropy (DLS) are localized in the same parts of the molecules and thus both methods monitor the same dynamics of the entire molecules.

\section{ACKNOWLEDGMENT}

Partial financial support of the Committee for Scientific Research, Poland (KBN, Grant No. 1 P03B 083 26) is gratefully acknowledged.

${ }^{1}$ C. A. Angell, K. L. Ngai, G. B. McKenna, P. F. McMillan, and S. W. Martin, Appl. Phys. Rev. 88, 3113 (2000).

${ }^{2}$ G. Meier, B. Gerharz, D. Boese, and E. W. Fischer, J. Chem. Phys. 94, 3050 (1990).

${ }^{3}$ A. Patkowski, M. Paluch, and H. Kriegs, J. Chem. Phys. 117, 2192 (2002).

${ }^{4}$ Data for PDE: F. Stickel, E. W. Fischer, and R. Richert, J. Chem. Phys. 104, 2043 (1996); data for PDE and KDE: F. Stickel, F. Kremer, and E. W. Fischer, Physica A 201, 318 (1993); data for BMPC and BMMPC: F Stickel, Ph.D. thesis, University of Mainz (1995).

${ }^{5}$ M. Paluch, K. L. Ngai, and S. Hensel-Bielowka, J. Chem. Phys. 114, 10872 (2001).

${ }^{6}$ J. Gapiński, M. Paluch, and A. Patkowski, Phys. Rev. E 66, 011501 (2002).

M. Paluch, R. Casalini, A. Best, and A. Patkowski, J. Chem. Phys. 117, 7624 (2002).

${ }^{8}$ M. Paluch, C. M. Roland, R. Casalini, G. Meier, and A. Patkowski, J. Chem. Phys. 118, 4578 (2003).

${ }^{9}$ F. Alvarez, A. Hoffman, A. Alegria, and J. Colmenero, J. Chem. Phys. 105, 432 (1996)

${ }^{10}$ Sz. Vass, A. Patkowski, E. W. Fischer, K. Süvegh, and A. Vertes, Europhys. Lett. 46, 815 (1999).

${ }^{11}$ R. Boehmer, K. L. Ngai, C. A. Angell, and D. J. Plazek, J. Chem. Phys. 99, 4201 (1993)

${ }^{12}$ S. Havriliak and S. Negami, Polymer 8, 101 (1967).

${ }^{13}$ S. Kahle, K. Schröter, E. Hempel, and E. Donth, J. Chem. Phys. 111, 6462 (1999).

${ }^{14}$ C. H. Wang, G. Fytas, D. Lilge, and Th. Dorfmüller, Macromolecules 14, 1363 (1981).

${ }^{15}$ B. Berne and R. Pecora, Dynamic Light Scattering (Wiley, New York, 1976).

${ }^{16}$ G. Meier, F. Fujara, and W. Petry, Macromolecules 22, 4421 (1989).

${ }^{17}$ K. Schmidt-Rohr and H. W. Spiess, Multidimensional NMR and Polymers (Academic, New York, 1994).

${ }^{18}$ D. Boese, B. Momper, G. Meier, F. Kremer, J.-U. Hagenah, and E. W. Fischer, Macromolecules 22, 4416 (1989). 
${ }^{19}$ F. Alvarez, A. Alegria, and J. Colmenero, Phys. Rev. B 44, 7306 (1991).

${ }^{20}$ E. Rössler, M. Taupitz, K. Börner, M. Schulz, and H.-M. Vieth, J. Chem. Phys. 92, 5847 (1990).

${ }^{21}$ G. Diezemann and K. Nelson, J. Phys. Chem. B 103, 4089 (1999).
${ }^{22}$ M. A. Floriano and C. A. Angell, J. Chem. Phys. 91, 2537 (1989).

${ }^{23}$ B. Schiener and R. Böhmer, J. Non-Cryst. Solids 182, 180 (1995).

${ }^{24}$ C. Hansen, F. Stickel, T. Berger, R. Richert, and E. W. Fischer, J. Chem. Phys. 107, 1086 (1997). 